\title{
Visible-Light-Driven Reductive (Cyclo)Dimerization of Chalcones over Heterogeneous Carbon Nitride Photocatalyst
}

\author{
Bogdan Kurpil, Yevheniia Markushyna, and Aleksandr Savateev*(i) \\ Max-Planck Institute of Colloids and Interfaces, Department of Colloid Chemistry, Research Campus Golm, 14424 Potsdam, \\ Germany
}

\section{Supporting Information}

ABSTRACT: Single-electron reduction of chalcones to the respective radical anions is a useful technique to activate these molecules toward subsequent transformations. Herein, a metalfree photocatalytic version of chalcones reduction in the presence of triethanolamine as a convenient electron donor and using heterogeneous carbon nitride visible-light photocatalyst is presented. The reaction proceeds via a long-lived radical species of the heterogeneous organic semiconductor. The scope of the reaction was studied, and regioselectivity of the chalcone radicals coupling was investigated. (1) Ten chalcones gave selectively polysubstituted cyclopentanoles with $31-73 \%$ isolated yield; (2)

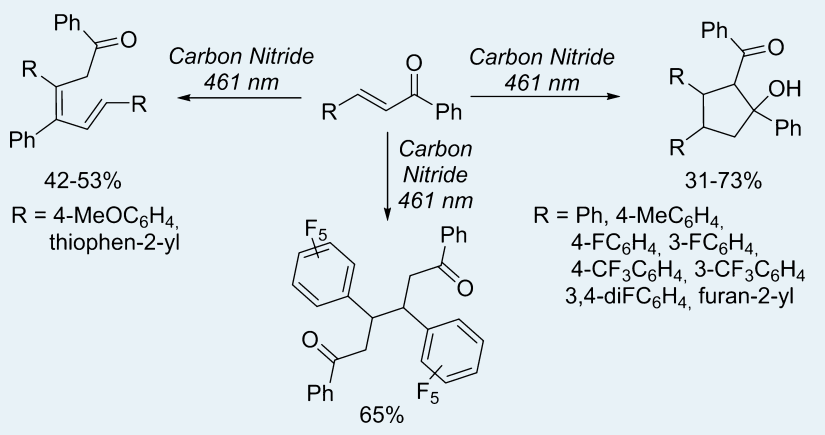
Two chalcones bearing electron-donor groups, $4-\mathrm{MeOC}_{6} \mathrm{H}_{4}$ and 2-thienyl, gave selectively the $\beta$-ketodienes in $42 \%$ and $53 \%$ isolated yield, respectively; (3) Pentafluorophenyl substituted chalcone gave exclusively the product of the radicals coupling followed by hydrogen transfer from triethanolamine-hexane-1,6dione in $65 \%$ isolated yield. Reductive cross cyclodimerization of a mixture of two different chalcones proceeded regioselectively with the formation of one product out of four possible. The mechanism was investigated by cyclic voltammetry and linear sweep voltammetry and suggests that the reaction proceeds through proton-coupled electron transfer.

KEYWORDS: organic photoredox catalysis, carbon nitride, chalcone, cyclopetanole, proton coupled electron transfer

$\mathrm{T}$ he rising demands to the efficacy of chemical processes prompt chemists to develop more performing and durable catalysts in order to enable challenging organic transformations with high selectivity, less energy input, and avoiding use of auxiliary reagents. A heterogeneous carbon nitride photocatalyst was originally developed for water splitting. ${ }^{1}$ However, in the past few years a new branchcarbon-nitride-based organic photoredox catalysis-that comprises a number of novel reactions has emerged. ${ }^{2,3}$ This is to be added to a number of carbon nitride materials with a stoichiometry $\mathrm{C}_{3} \mathrm{~N}_{x}$ and mesoporous carbon nitrides with developed surface area reported by Vinu et al., ${ }^{4,5}$ Carbon nitride materials are applied in different areas including, for example, hydrogen production, ${ }^{6} \mathrm{CO}_{2}$ sorption and conversion, and organocatalysis. ${ }^{7-9}$

Cyclopentane moiety occurs often in many biologically active commercial compounds (Scheme 1a). ${ }^{10-14}$ Scheme $1 \mathrm{~b}$ briefly summarizes available methods of cyclopentane core construction. Most of these methods are based on cyclization of $\omega$-functionalized alkynes, ${ }^{15-17} \alpha, \omega$-enynes, ${ }^{18,19}$ or $\alpha, \omega$ dienes. $^{20,21}$ These methods are considered as primary for simple cyclopentanes. However, if heavily substituted cyclopentanes are of interest, synthesis of the respective precursors (e.g., substituted $\alpha, \omega$-enynes) might be a challenging multistep process.
Alternatively, reductive cyclodimerization of chalcones, readily available from the corresponding aldehydes and ketones, offers a route to polysubstituted cyclopentanes just in one step. ${ }^{22-27}$ This reaction typically requires transition metal salts taken in equimolar ratio with respect to the substrate and allows for homocoupling of chalcones only. Earlier Xia and Zhao reported that homocoupling of chalcones can be also achieved by combining homogeneous $\mathrm{Ru}$ $(\mathrm{bpy})_{3}\left(\mathrm{PF}_{6}\right)_{2}$ photocatalyst in the presence of $\mathrm{Sm}(\mathrm{OTf})_{2}{ }^{27}$

Given our own results in developing new organic photoredox reactions, for example, synthesis of 1,3,4-oxadiazoles, ${ }^{28}$ photocatalytic Kindler reaction and Hantzsch pyridine synthesis, ${ }^{29,30}$ oxidative $\mathrm{C}-\mathrm{H}$ thiolation of toluenes, ${ }^{31}$ as well as reciting a number of reports from Blechert and Wang, such as trifluoromethylation of heterocycles, ${ }^{17}$ oxidative coupling of benzylamines, $^{32}$ and selective oxidation of alcohols and hydrocarbons, ${ }^{33,34}$ all mediated by carbon nitrides, we assume this class of materials to be effective for the reductive dimerization of chalcones. Using heterogeneous photocatalyst, we can benefit from recyclability and high stability of this material. As a result, regioselectivity and therefore cross

Received: October 17, 2018

Revised: January 15, 2019

Published: January 17, 2019 
Scheme 1. Overview of Cyclopentanoles and Cyclopentanes

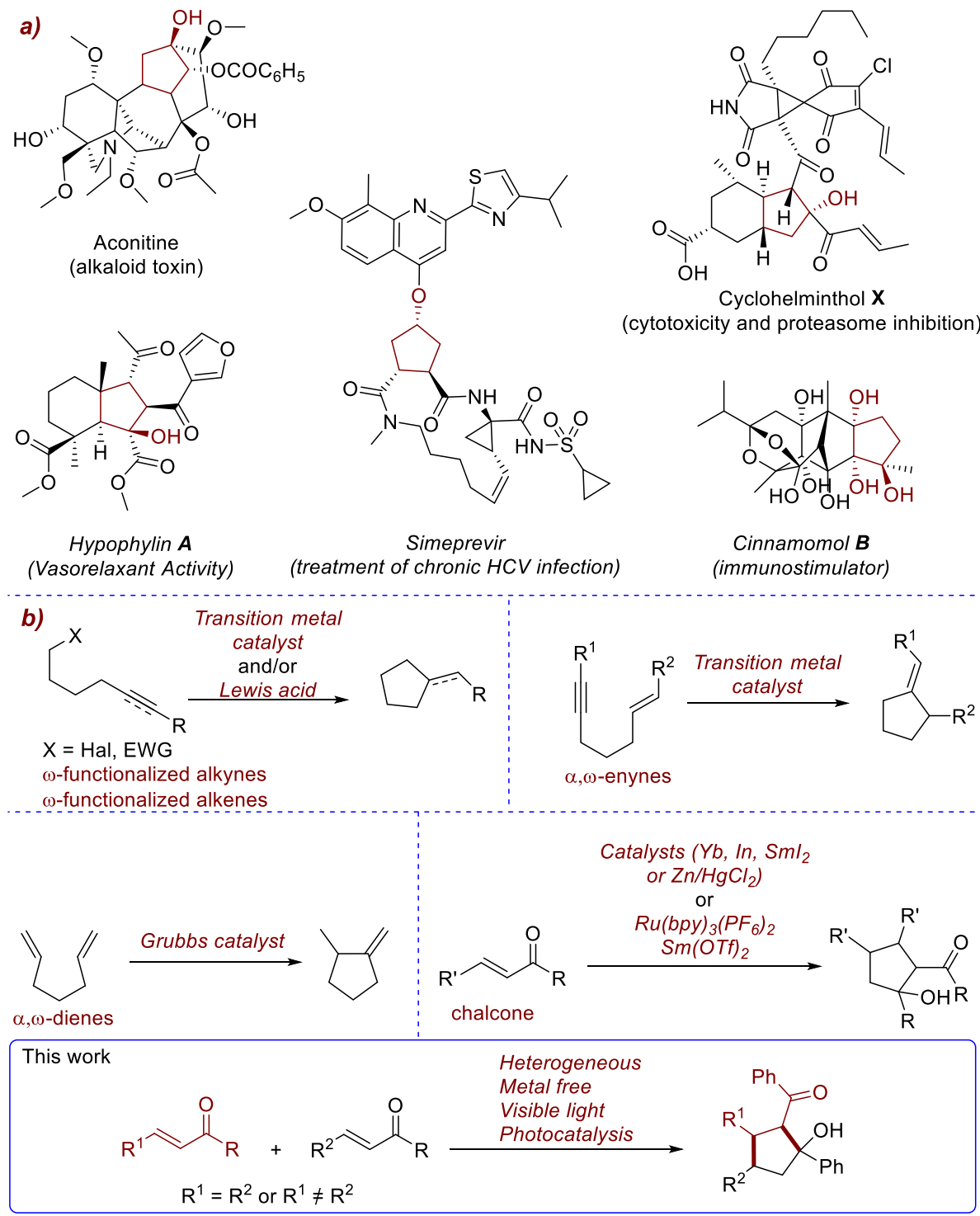

${ }^{a}$ Selected examples of biologically active cyclopentanole derivatives; ${ }^{b}$ Known approaches to synthesis of cyclopentanes and the concept of this work.

cyclodimerization of chalcones, because of coordination of the substrate to the photocatalyst surface, can be achieved.

Among the variety of carbon nitride materials especially useful for organic photoredox applications are those bearing negatively charged nitrogen atoms, for example, poly(heptazine imides) (Figure 1) reported by Dontsova et. al, ${ }^{35}$ or cyanamide-functionalized carbon nitrides reported by Lotsch et al. ${ }^{36}$ The unique feature of these materials is to "trap" electrons and to give a long-lived radical anion, ${ }^{31,37-39}$ which can be directly observed as green or deep-blue solids. They might also be beneficial for single-electron reduction of chalcones in order to generate the radical of chalcones and hence to trigger their coupling.

In this work, we present a new visible-light-driven metal-free methodology for the single-electron reduction of chalcones to the respective radicals using potassium poly(heptazine imide) (K-PHI) as a heterogeneous photocatalyst without using any coordinative additives. Cross reductive cyclodimerization is implemented by using advantage of the heterogeneous
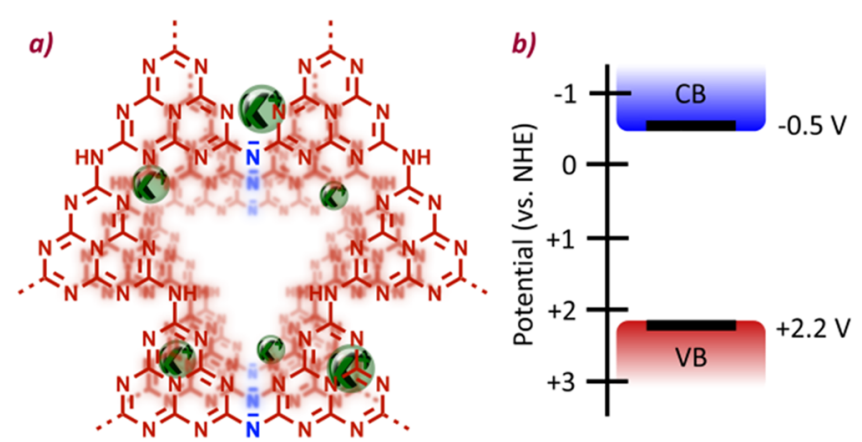

Figure 1. K-PHI chemical (a) and band (b) structure.

photocatalysis (Scheme 1b). Several hexa-3,5-dien-1-ones or hexane-1,6-diones are readily available via this method as well.

The photocatalyst, K-PHI, was synthesized according to the described procedure, and its characteristics are given in Figure S2. ${ }^{30}$ Photocatalytic reductive transformation of chalcone 1a, 
using triethanolamine (TEOA) as electron donor, was chosen as the initial reaction for study (Table 1). In the control

Table 1. Screening of the Reaction Conditions in Photocatalytic Formation of Cyclopentanole $2 \mathrm{a}^{a}$

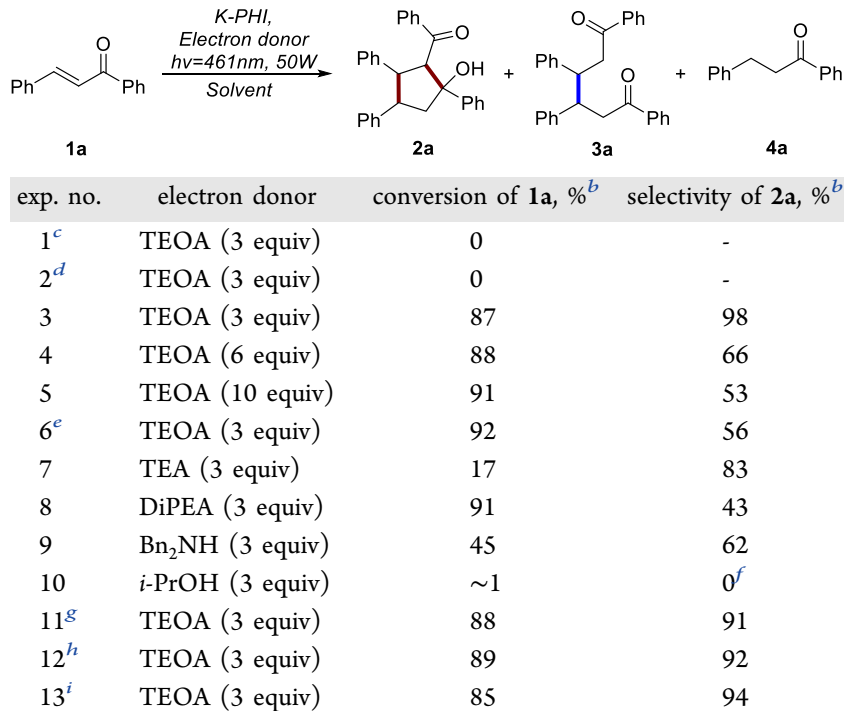

${ }^{a}$ Reaction conditions: chalcone $(50 \mu \mathrm{mol})$, electron donor, K-PHI $(10 \mathrm{mg})$, acetonitrile $(2 \mathrm{~mL}), 80^{\circ} \mathrm{C}, 20 \mathrm{~h}, \lambda=461 \mathrm{~nm}$, under argon atmosphere. ${ }^{b}$ Conversion of $1 \mathrm{a}$ and selectivity for $2 \mathrm{a}$ were determined by GC-MS. ${ }^{c}$ Without photocatalyst. ${ }^{d}$ Without light irradiation. ${ }^{e} 25$ ${ }^{\circ} \mathrm{C}, 60$ h. ${ }^{f_{\text {Trace }}}$ amounts of $3 \mathrm{a}$ were observed. ${ }^{g}$ Second run. ${ }^{h_{\mathrm{T}}}$ Third run. ${ }^{i}$ Fourth run.

reactions without the photocatalyst (entry 1) or without light irradiation (entry 2), no products were formed. Under light irradiation $\left(\lambda=461 \mathrm{~nm}, 51.7 \pm 0.03 \mathrm{~mW} \mathrm{~cm}^{-2}\right.$, Blue LED $)$ with K-PHI as the photocatalyst, three products formed: cyclopentanole $\mathbf{2 a}$, reduced dimer $\mathbf{3 a}$, and ketone $\mathbf{4 a}$, which is the product of the starting chalcone reduction. GC-MS also revealed that during the reaction, TEOA lost the $\mathrm{H}_{2}$ molecule. The highest selectivity (98\%) of the desirable cyclopentanole 2a, with high conversion ( $87 \%$ ) of the chalcone, was obtained after $20 \mathrm{~h}$ of the reaction at $80{ }^{\circ} \mathrm{C}$ using 3 equiv of TEOA in acetonitrile as a solvent (entry 3 ). Increasing the excess of TEOA (entries 4-5) led to slightly higher conversion (8891\%) of the chalcone, but at the same time provided significant amount of the dimer 3a, thereby decreasing the selectivity of the cyclopentanole formation (53-66\%). Lower selectivity toward $2 \mathrm{a}(56 \%)$ was observed at room temperature (entry 6), which proves the prevalence of the thermodynamic control in the formation of the five-membered ring. Other amines as electron donors (entries 7-9) are less effective and gave lower conversions and/or lower selectivity. In case of iso-propanol, as the electron donor (entry 10), only traces of the dimer 3a were formed in this reaction. The activity of the photocatalyst was almost the same even after four times of the catalyst recycling (entries 11-13), which proves good stability of K-PHI under the photocatalytic conditions.

Using the optimized reaction conditions, cyclopentanoles $\mathbf{2 a}-\mathbf{g}$ were synthesized and isolated in good yields (Scheme 2). Slightly higher yields were observed for cyclopentanoles $\mathbf{2} \mathbf{c}-\mathbf{g}$ substituted with fluorine atoms in the aromatic ring. The presence of fluorine reduces reduction potential of chalcones $\mathbf{1 c}-\mathbf{g}$ compared with the compounds $\mathbf{1 a}-\mathbf{b}$ and therefore facilitates reductive cyclodimerization. Chalcone $\mathbf{1 h}$, which
Scheme 2. Scope of Cyclopentanoles $2 a-h$ Synthesized via a Photocatalytic Reductive Cyclodimerization of Chalcones ${ }^{a}$
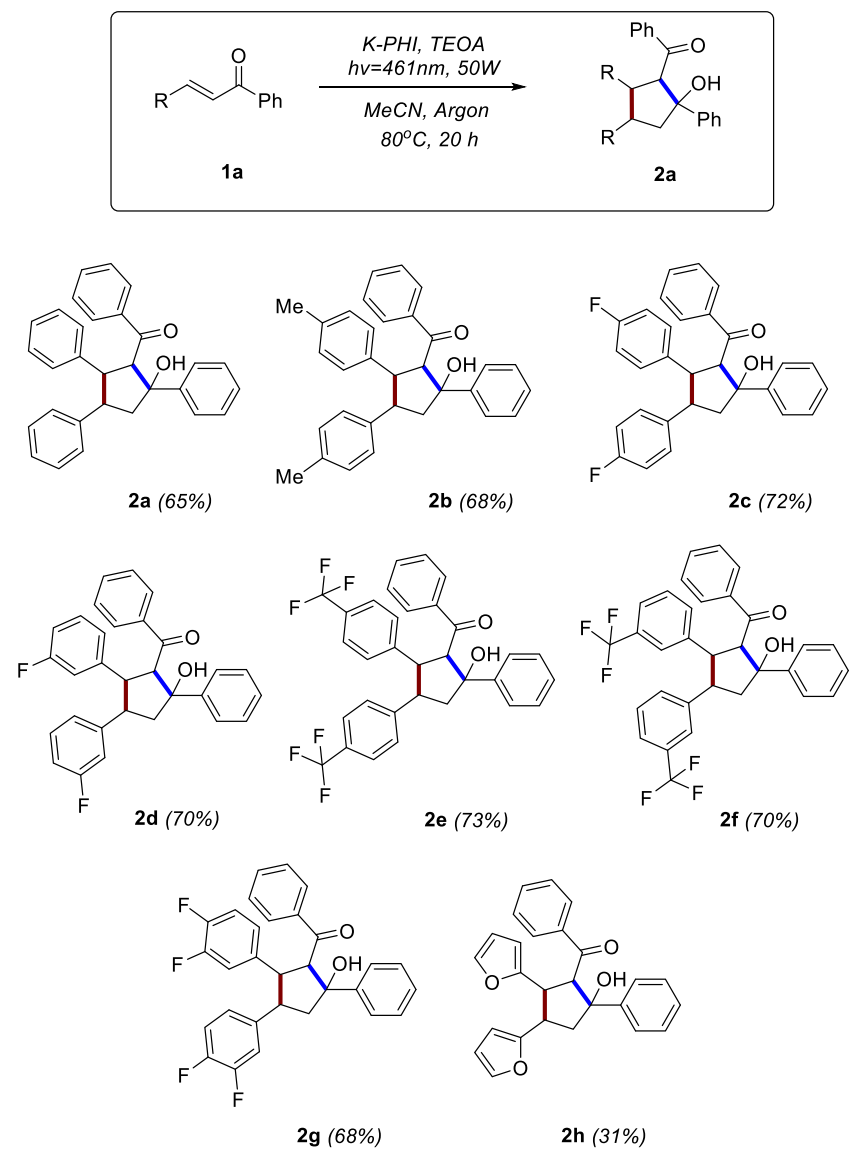

${ }^{a}$ Isolated yields are shown in parentheses.

contains electron-donor furyl moiety, gave the corresponding cyclopentanole $\mathbf{2 h}$ along with a large amount of highmolecular-weight undefined compounds. From this mixture, the product $\mathbf{2 h}$ was isolated with only $31 \%$ yield. Such result could be explained by the high impact of the electron-donor character of the substituent on the reaction path (see below explanation of the mechanism). In general the isolated yields are lower than the yields determined by GC-MS. It is related to the difficulty of the product separation in the presence of large amount of TEOA oxidation products using column chromatography.

We also attempted to couple chalcones bearing methyl substituent at the carbonyl group, such as (E)-4-phenylbut-3en-2-one, 1-(cyclohex-1-en-1-yl)ethan-1-one and cyclohex-2en-1-one as well as methyl cinnamate. However, none of these enones gave cyclopentanoles. The explanation originates from the significantly more negative reduction potentials of the aliphatic chalcones $\left(E_{\text {red }}=-1.7 \text { to }-2.2 \mathrm{~V} \text { vs SCE }\right)^{40}$ and methyl cinnamate $\left(E_{\mathrm{red}}=-1.9 \mathrm{~V}\right.$ vs SCE $),{ }^{41}$ due to worse resonance stabilization of the intermediary radicals, compared with aromatic ones (chalcone $1 \mathrm{a}, E_{\text {red }}=-1.4 \mathrm{~V}$ vs SCE). ${ }^{42} \mathrm{On}$ the other hand, coordination of the substrate to the surface of the photocatalyst is essential for the electron transfer. This interaction is more efficient in case of planar aromatic chalcones rather than aliphatic ones. All in all, the method works well for (het)aryl-substituted chalcones and even better for arylsubstituted chalcones with electron-withdrawing groups due to efficient stabilization of the intermediates. 
In order to check if cross cyclodimerization can be achieved using K-PHI, we mixed chalcones $\mathbf{1 a}$ and $\mathbf{1 c}$ in one pot in equimolar ratio (Scheme 3 ). In this case, we were also pleased

Scheme 3. Cross Reductive Coupling of Chalcones ${ }^{a}$

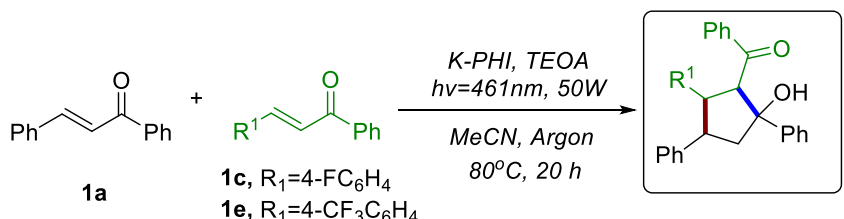

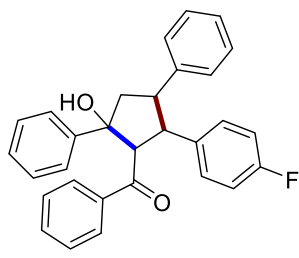

2 ac $(42 \%)$

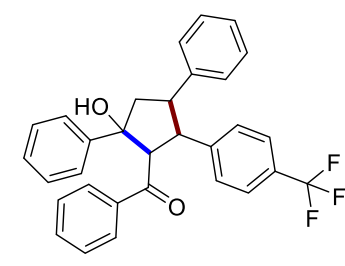

2ae (45\%)
${ }^{a}$ Isolated yields are shown in parentheses.

to observe formation of unsymmetrically substituted cyclopentanole 2ac. After purification, it was isolated with $42 \%$ yield. Similarly chalcones 1 a and 1 e gave the product 2 ae with $45 \%$ yield. These results were obtained under nonoptimized conditions. Notably, the yields of chalcones 2ac and 2ae are higher than their content in the theoretical mixture due to statistical distribution of the possible products (25\%). In addition, in HR-MS of the reaction mixture we have not observed other cyclopentanoles derived from homocoupling of two chalcones (i.e., compounds $2 \mathbf{a}, \mathbf{2 c}$ ). All this implies the existence of regio-control in the photocatalytic reaction.

Behavior of the chalcones $\mathbf{1} \mathbf{i}-\mathbf{k}$ under the conditions of photocatalysis (Scheme 4) drastically differs from other described above. Chalcones $\mathbf{1} \mathbf{i}, \mathbf{j}$ gave a mixture of cyclopentanoles $\mathbf{2} \mathbf{i}, \mathbf{j}$ and dienone $\mathbf{5 i} \mathbf{i} \mathbf{j}$ respectively, with the predominance of the dienone product (with ratio $5 \mathbf{i}: 2 \mathbf{i}$ of $3: 1$ and $5 \mathbf{j}: \mathbf{2} \mathbf{j}$ of $2: 1)$. Important to note, dienones $\mathbf{5}$ were not reported earlier. Synthesis of these compounds using a toolbox of traditional organic chemistry presumably would require

Scheme 4. Transformation of Chalcones $1 \mathrm{i}-\mathrm{k}$ under Photocatalytic Conditions ${ }^{a}$

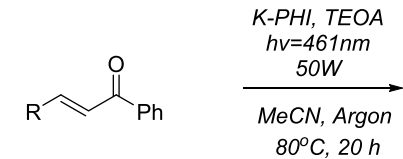

1i, $\mathrm{R}=4-\mathrm{MeOC}_{6} \mathrm{H}_{4}$ 1j, $R=2$-Thienyl

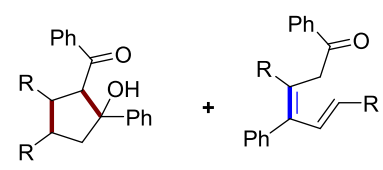

$2 i(18 \%)$
$2 j(22 \%)$
$5 i(53 \%)$ $5 j(42 \%)$

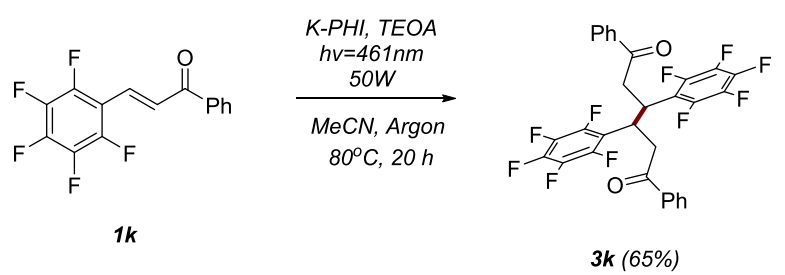

${ }^{a}$ Isolated yields are shown in parentheses. several steps, while in the present method they are prepared in one step from the corresponding chalcones, though in moderate yield.

These results may be explained by the two competitive reaction pathways, involving two different reactive centers in the starting chalcone: double carbon-carbon bond and carbonyl group (see below explanation of the mechanism). Formation of the dimer $\mathbf{3 k}$ as the single product for chalcone with pentafluorophenyl substituent evidently comes from higher thermodynamic stability of the linear structure. It keeps bulky pentafluorophenyl substituents far from each other due to a free rotation around a single carbon-carbon bond. Therefore, steric hindrance is minimal, compared with the restricted cyclic geometry.

The kinetic data of the chalcone 1a conversion over time, as shown in Figure 2a, suggest that there is an induction period of

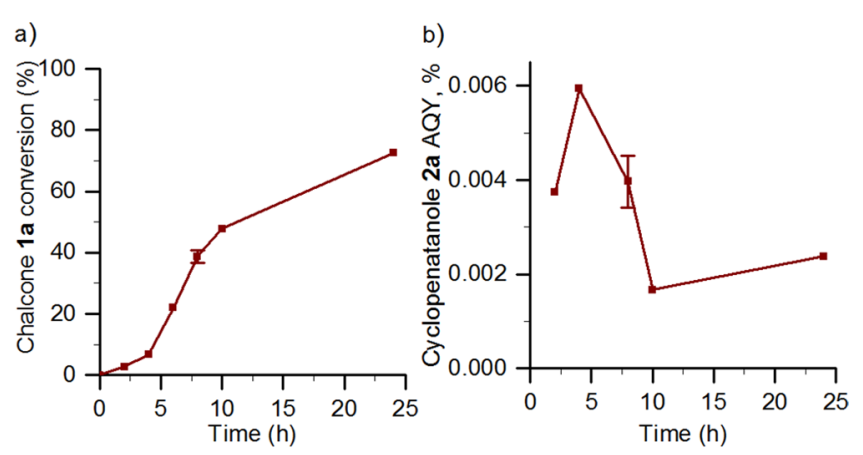

Figure 2. (a) Time-dependent conversion of the chalcone 1a. (b) Time-dependent AQY of the chalcone $2 \mathrm{a}$.

ca. $4 \mathrm{~h}$ during in which the reaction proceeds slower. The induction period is explained by the accumulation of the longlived K-PHI radical anion, that is, charging of K-PHI (see mechanism discussion below). The apparent quantum yield (AQY) of the cyclopentanole $\mathbf{2 a}$ is $0.005 \%$, while the relative error of AQY determination is $14 \%$.

In order to get insight into the redox properties of the species employed in the photocatalytic reaction, we performed a series of electrochemical measurements in acetonitrile using $(n-\mathrm{Bu})_{4} \mathrm{~N}^{+} \mathrm{ClO}_{4}^{-}(0.1 \mathrm{M})$ as a supporting electrolyte. Figure $3 \mathrm{a}$ shows the $\mathrm{CV}$ curves of different components of the reaction mixture. $\mathrm{MeCN}$ is stable on the reduction side at least up to $-2.2 \mathrm{~V}$ vs $\mathrm{Ag} / \mathrm{AgNO}_{3}(0.01 \mathrm{M})$ and on the oxidation side at least up to $+1.8 \mathrm{~V}$. As expected for amines, TEOA has the onset of oxidation $+0.34 \mathrm{~V}$, while it is stable against reduction up to $-1.9 \mathrm{~V}$. Oxidation of 1 a starts at $+1.6 \mathrm{~V}$, while reduction at $-1.7 \mathrm{~V}$. When excess of TEOA was added to $1 \mathrm{a}$, the resultant mixture still had the onset of oxidation at $+0.34 \mathrm{~V}$, suggesting that in the presence of $1 \mathbf{a}$ in the photocatalytic experiment, TEOA is still more likely to act as electron donor. However, the reduction potential of 1 a was shifted to $-1.4 \mathrm{~V}$. It might be explained by proton-coupled electron transfer (PCET), assuming that intermediary $[\text { TEOA }]^{\bullet+}$ is acidic enough to serve as a proton donor. In agreement, the CV curve obtained for 1a in the presence of $\mathrm{HCl}(4 \mathrm{mM})$, which is an even better proton donor compared with $[\text { TEOA }]^{\bullet+}$. In this case, the onset of the reduction current was observed at -0.5 $\mathrm{V}$.

Figure $3 \mathrm{~b}$ represents $\mathrm{CV}$ of chalcones 1a,c,e. Thus, $p-\mathrm{F}$ substituted chalcone $1 \mathrm{c}$ has the reduction potential of $-1.7 \mathrm{~V}$. $p-\mathrm{CF}_{3}$ substituted chalcone $1 \mathrm{e}$ has more positive potential of 

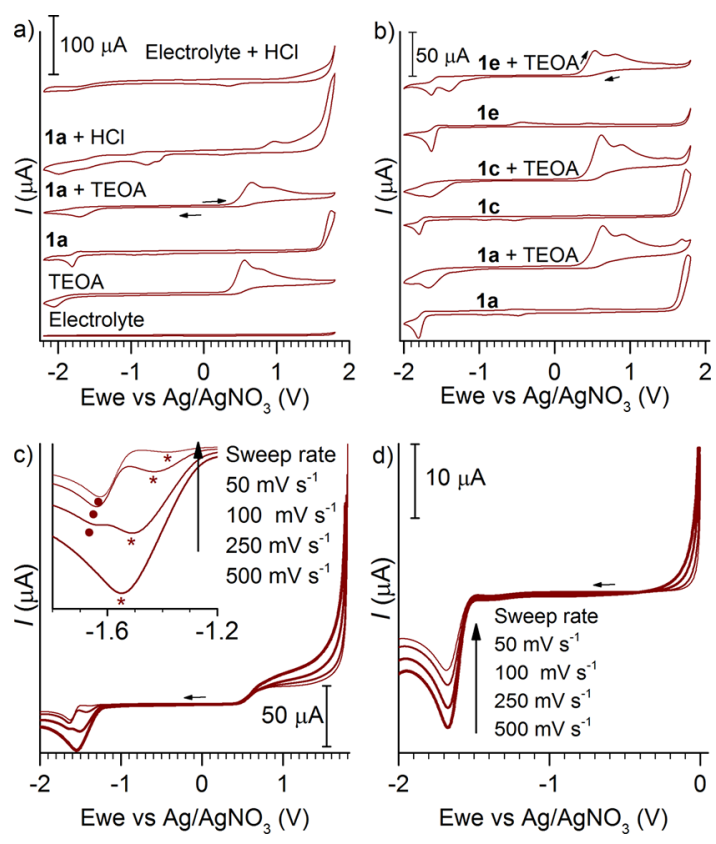

Figure 3. Electrochemical study. Three-electrode cell was used: glassy carbon (WE), $\mathrm{Ag} / \mathrm{AgNO}_{3}$ in acetonitrile (RE), Pt wire (CE). (a) Cyclic voltammetry (CV) curves were acquired at a scan rate $250 \mathrm{mV}$ $\mathrm{s}^{-1}$. Direction of scanning is shown with arrows and is the same for all CVs. Second cycle is shown. (b) CVs of a mixture of the chalcones $(2.2 \mathrm{mM})$ and TEOA $(7.6 \mathrm{mM})$ measured at $250 \mathrm{mV} \mathrm{s}^{-1}$. (c) Linear sweep voltammetry (LSV) of $1 \mathrm{e}(2.2 \mathrm{mM})$ measured at different sweep rates. Inset shows the magnified region of the LSV. (d) LSV of 1e $(2.2 \mathrm{mM})$ and TEOA $(7.6 \mathrm{mM})$ measured at different sweep rates.

$-1.5 \mathrm{~V}$. These findings agree with the Hammett constants of the above-mentioned groups $-\sigma_{\mathrm{p}}(\mathrm{F})=0.06$ and $\sigma_{\mathrm{p}}\left(\mathrm{CF}_{3}\right)=$ $0.54 .^{43}$ In the presence of TEOA $(7.6 \mathrm{mM})$ the onset of the reduction current, for all chalcones, presumably due to PCET, was observed at lower overpotential, in the range from $-1.1 \mathrm{~V}$ to $-0.9 \mathrm{~V}$.

Figure 3c shows LSV curves recorded for chalcone 1e at different sweep rates. At high sweep rate, $\geq 500 \mathrm{mV} \mathrm{s}^{-1}$, only the reduction peak at $-1.55 \mathrm{~V}$ (marked with "star") is observed. When the sweep rate was decreased to $250 \mathrm{mV} \mathrm{s}^{-1}$, an additional peak (marked with "filled circles") at $-1.65 \mathrm{~V}$ appeared. By decreasing the sweep rate, the intensity of the former peak decreased while the later became more pronounced. The peak at the potential $-1.65 \mathrm{~V}$ became dominant at the sweep rate of $50 \mathrm{mV} \mathrm{s}^{-1}$. The onset of the reduction current in this case is $-1.53 \mathrm{~V}$, which is close to the one observed in the absence of TEOA (Figure 3b). Finally, when only the reduction region from $0 \mathrm{~V}$ to $-2.2 \mathrm{~V}$ was scanned, regardless of the sweep rate, 1e had onset of reduction at $-1.5 \mathrm{~V}$ (Figure $3 \mathrm{~d}$ ).

These results imply that PCET assisted by proton transfer from $[\mathrm{TEOA}]^{\bullet+}$ may be observed in the electrochemical cell at high sweep rate. On the other hand, in the photoreactor, the photocatalyst provides both reduction and oxidation sites at the same time. Therefore, PCET may be envisioned as a major process in the photocatalytic reductive cyclodimerization of the chalcones.

Taking into account the results of CV and LSV, the formation of the reductive dimerization products-cyclopentanoles 2, dienones 5 and reduced dimers 3 could be explained by the following mechanism (Scheme 5). Excited under the visible light K-PHI* photocatalyst promptly removes an electron from TEOA (electron donor) and converts into long-lived radical-anion $\mathrm{K}-\mathrm{PHI}^{\bullet-}$ that is observed as deepgreen solid (inset on the Scheme 5), reported earlier. ${ }^{31,37,38}$ The chalcone $\mathbf{1}$ is reduced to the radical 7 via PCET. In this process, $\mathrm{K}-\mathrm{PHI}^{\bullet-}$ donates an electron, while $[\mathrm{TEOA}]^{\bullet+}$ serves as a proton donor.

On the basis of the experimental data (Table 1, Scheme 2,4), the tentative intermediate 7 can undergo four reaction paths. First, it can be reduced via PCET by the intermediate 6 to the ketone 4 (Path A).

On an important note, we could not identify the exact structure of the final product of TEOA oxidation. However, from the GC-MS of the reaction mixture we deduced that TEOA lost $\mathrm{H}_{2}$ molecule. On the Scheme 5 the product of TEOA oxidation is denoted as $\left[\mathrm{TEOA}-\mathrm{H}_{2}\right]$. A discussion about plausible structure of $\left[\mathrm{TEOA}-\mathrm{H}_{2}\right]$ is given in the Supporting Information.

Second, intermediate 7 reacts with a molecule of starting chalcone 1 . Addition to the C-3 carbon atom of the chalcone 1 followed by SET from 6 leads to the intermediate 9. This intermediate can be converted into the reduced dimer 3 (Path B). Pentafluorophenyl substituents in $\mathbf{1 k}$ apparently stabilize the intermediate 9 and make cyclization to the respective cyclopentanole unfavorable. A small amount of the reduced dimer 3a was also observed when chalcone 1a was taken as a substrate. In agreement with this reaction path, a large excess of TEOA (Table 1, entry 4,5) facilitates formation of the reduced dimer 3 and as a result decreases the yield of the cyclopentanole $\mathbf{2 a}$.

Third, intramolecular aldol condensation of 9 gives cyclopentanole 2 (Path $\mathrm{C}$ ). Path $\mathrm{C}$ is preferential for most of the chalcones.

Finally, addition of the radical 7 to the C-1 carbon atom of the chalcone $\mathbf{1}$ followed by SET leads to the intermediate 10, that is converted to the compound $\mathbf{1 1}$ upon proton transfer from 8 (Path D). In case of the chalcones $\mathbf{1} \mathbf{i}, \mathbf{j}$, electron-donor substituents in the aromatic ring increase the electron density on the double bond. An electron-rich substituent alters the reactivity of the radical-anion 7 and directs the attack at the carbonyl group (electrophilic center). Thermally induced elimination of $\mathrm{H}_{2} \mathrm{O}$ molecule produces dienone 5. Path $\mathrm{D}$ is preferential for the chalcones $\mathbf{2} \mathbf{i}, \mathbf{j}$, bearing electron-rich groups.

The mechanism of cyclopentanol formation (Path C) is additionally supported by the results of the cyclopentanoles 2ac and 2ae synthesis from a mixture of chalcone 1a and chalcone 1c or 1e. Because of the presence of electronwithdrawing $p-\mathrm{F}$ or $p-\mathrm{CF}_{3}$ substituents, reduction of chalcones 1c and 1e is more favorable compared with the chalcone 1 a. The radicals, $7 \mathrm{c}$ and $7 \mathbf{e}$, are then added to the double bond of the chalcone 1a, but not vice versa. Therefore, the electronic structure of the chalcones in this reaction dictates the regioselectivity of the products formation.

Having the scheme of the mechanism, relatively low AQY may be rationalized by the multistep nature of cyclopentanole synthesis. Thus, formation of the key radical intermediate 7 requires stoichiometric amount of photons that first must be absorbed by the photocatalyst. Second, recombination of the photogenerated holes and electrons would apparently decrease the AQY of the whole process. Furthermore, at least three competing processes, for the intermediate $7 \mathbf{a}$, exist along with the primary reaction of the cyclopentanole $\mathbf{2 a}$ formation. 
Scheme 5. Proposed Mechanism of the Photocatalytic Transformation of Chalcones ${ }^{a}$

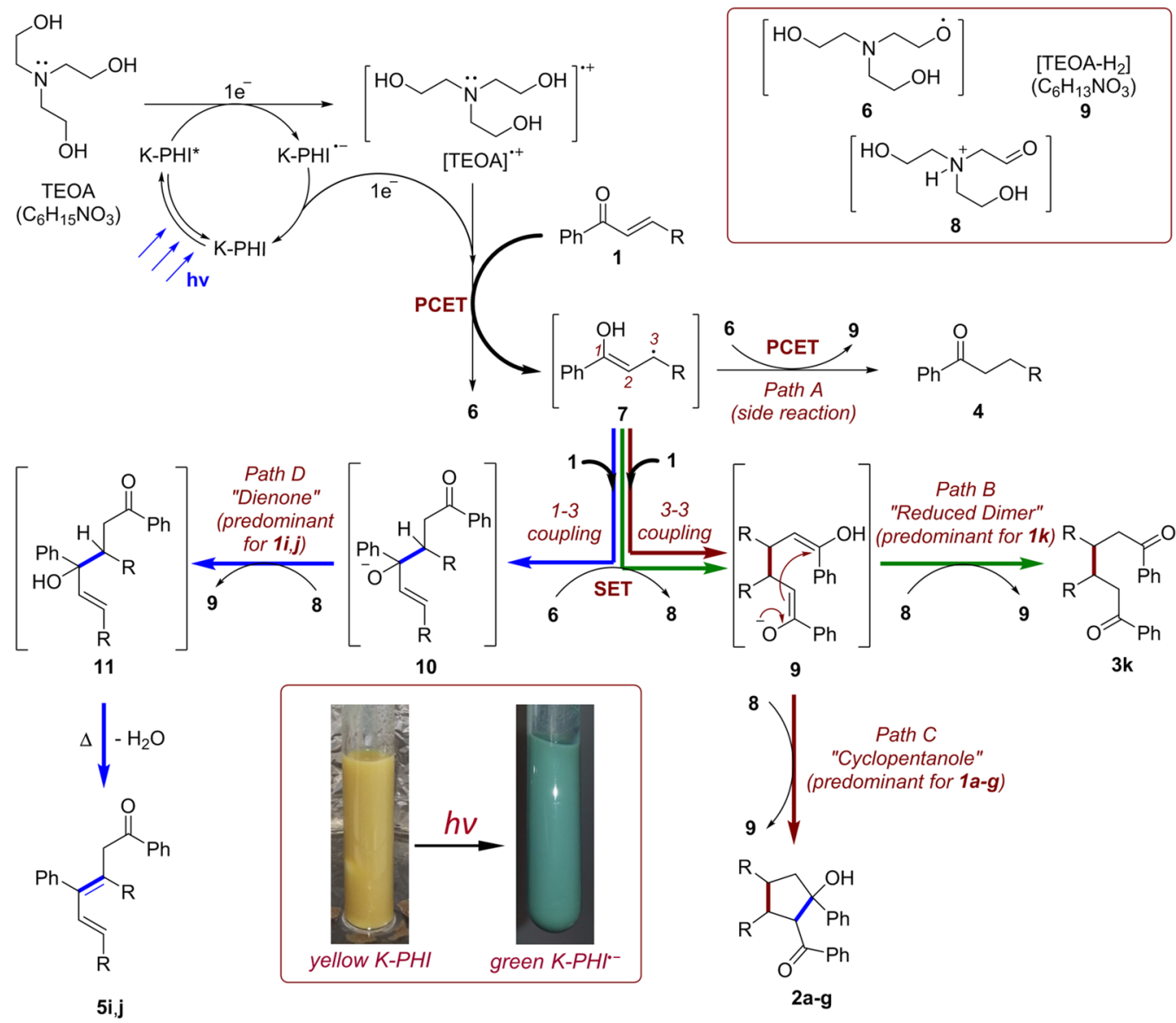

${ }^{a}$ Appearance of the reaction mixture before irradiation (yellow suspension) and after irradiation (green suspension) is shown in inset at the bottom of the scheme. Possible structures of the intermediates and the product of TEOA oxidation are shown in top right rectangle.

Finally, dead ends, that is, quenching of the radical intermediates (e.g., 7) cannot be excluded.

In summary, the photocatalytic methodology of reductive dimerization of chalcones under visible light irradiation using a noble-metal-free, heterogeneous photocatalyst, potassium poly(heptazine imide), was developed. Cyclopentanoles were obtained in good yields as major products for the majority of substrates. Chalcones bearing electron-donor aromatic and heteroaromatic groups converted preferably to dienonesalternative products of the reductive dimerization. Presence of the bulky pentafluorophenyl substituent in the starting chalcone leads to the formation of the symmetric linear dimer as the single product, which is less sterically restrained, compared with the ring structure. The discovered photocatalytic transformation reveals a new simple approach for the dimerization of the double bond and is promising as a convenient tool for a modern preparative organic synthesis. The studied here reaction confirms the existence of regioselectivity in heterogeneous photocatalysis versus homogeneous version.

\section{ASSOCIATED CONTENT}

\section{S Supporting Information}

The Supporting Information is available free of charge on the ACS Publications website at DOI: 10.1021/acscatal.8b04182.
Experimental procedures, characterization data for photocatalyst and all synthesized chalcones and cyclopentanoles (PDF)

\section{AUTHOR INFORMATION}

\section{Corresponding Author}

*E-mail: oleksandr.savatieiev@mpikg.mpg.de.

\section{ORCID}

Aleksandr Savateev: 0000-0002-5760-6033

Funding

Deutsche Forschungsgemeinschaft (Grant Number An 156 131).

\section{Notes}

The authors declare no competing financial interest.

\section{ACKNOWLEDGMENTS}

The authors are grateful the Deutsche Forschungsgemeinschaft for the financial support (DFG-An 156 13-1) and Prof. Markus Antonietti for fruitful discussion.

\section{REFERENCES}

(1) Wang, X.; Maeda, K.; Thomas, A.; Takanabe, K.; Xin, G.; Carlsson, J. M.; Domen, K.; Antonietti, M. A Metal-Free Polymeric Photocatalyst for Hydrogen Production from Water under Visible Light. Nat. Mater. 2009, 8, 76-80. 
(2) Savateev, A.; Ghosh, I.; König, B.; Antonietti, M. Photoredox Catalytic Organic Transformations Using Heterogeneous Carbon Nitrides. Angew. Chem., Int. Ed. 2018, 57, 15936-15947.

(3) Savateev, A.; Antonietti, M. Heterogeneous Organocatalysis for Photoredox Chemistry. ACS Catal. 2018, 8, 9790-9808.

(4) Kim, I. Y.; Kim, S.; Jin, X.; Premkumar, S.; Chandra, G.; Lee, N.S.; Mane, G. P.; Hwang, S.-J.; Umapathy, S.; Vinu, A. Ordered Mesoporous $\mathrm{C}_{3} \mathrm{n}_{5}$ with a Combined Triazole and Triazine Framework and Its Graphene Hybrids for the Oxygen Reduction Reaction (Orr). Angew. Chem., Int. Ed. 2018, 57, 17135-17140.

(5) Lakhi, K. S.; Park, D.-H.; Al-Bahily, K.; Cha, W.; Viswanathan, B.; Choy, J.-H.; Vinu, A. Mesoporous Carbon Nitrides: Synthesis, Functionalization, and Applications. Chem. Soc. Rev. 2017, 46, 72101.

(6) Zhu, Y.; Marianov, A.; Xu, H.; Lang, C.; Jiang, Y. Bimetallic Ag$\mathrm{Cu}$ Supported on Graphitic Carbon Nitride Nanotubes for Improved Visible-Light Photocatalytic Hydrogen Production. ACS Appl. Mater. Interfaces 2018, 10, 9468-9477.

(7) Lakhi, K. S.; Park, D.-H.; Singh, G.; Talapaneni, S. N.; Ravon, U.; Al-Bahily, K.; Vinu, A. Energy Efficient Synthesis of Highly Ordered Mesoporous Carbon Nitrides with Uniform Rods and Their Superior $\mathrm{Co}_{2}$ Adsorption Capacity. J. Mater. Chem. A 2017, 5, $16220-16230$

(8) Park, D.-H.; Lakhi, K. S.; Ramadass, K.; Kim, M.-K.; Talapaneni, S. N.; Joseph, S.; Ravon, U.; Al-Bahily, K.; Vinu, A. Energy Efficient Synthesis of Ordered Mesoporous Carbon Nitrides with a High Nitrogen Content and Enhanced $\mathrm{Co}_{2}$ Capture Capacity. Chem. - Eur. J. 2017, 23, 10753-10757.

(9) Naidu Talapaneni, S.; Ramadass, K.; Ruban, S. J.; Benzigar, M.; Lakhi, K. S.; Yang, J.-H.; Ravon, U.; Albahily, K.; Vinu, A. 3d Cubic Mesoporous C3n4 with Tunable Pore Diameters Derived from Kit-6 and Their Application in Base Catalyzed Knoevenagel Reaction. Catal. Today 2018, DOI: 10.1016/j.cattod.2018.08.003.

(10) Rosenquist, Å.; Samuelsson, B.; Johansson, P.-O.; Cummings, M. D.; Lenz, O.; Raboisson, P.; Simmen, K.; Vendeville, S.; de Kock, H.; Nilsson, M.; Horvath, A.; Kalmeijer, R.; de la Rosa, G.; BeumontMauviel, M. Discovery and Development of Simeprevir (Tmc435), a Hcv Ns3/4a Protease Inhibitor. J. Med. Chem. 2014, 57, 1673-1693. (11) Zhou, L.; Tuo, Y.; Hao, Y.; Guo, X.; Tang, W.; Xue, Y.; Zeng, J.; Zhou, Y.; Xiang, M.; Zuo, J.; Yao, G.; Zhang, Y. Cinnamomols a and B, Immunostimulative Diterpenoids with a New Carbon Skeleton from the Leaves of Cinnamomum Cassia. Org. Lett. 2017, 19, 30293032.

(12) Wu, X.-D.; Luo, D.; Tu, W.-C.; Deng, Z.-T.; Chen, X.-J.; Su, J.; Ji, X.; Zhao, Q.-S. Hypophyllins a-D, Labdane-Type Diterpenoids with Vasorelaxant Activity from Hypoestes Phyllostachya "Rosea. Org. Lett. 2016, 18, 6484-6487.

(13) Chan, T. Y. K. Aconite Poisoning. Clin. Toxicol. 2009, 47, 279285.

(14) Tanaka, S.; Honmura, Y.; Uesugi, S.; Fukushi, E.; Tanaka, K.; Maeda, H.; Kimura, K.-i.; Nehira, T.; Hashimoto, M. Cyclohelminthol $\mathrm{X}$, a Hexa-Substituted Spirocyclopropane from Helminthosporium Velutinum Yone96: Structural Elucidation, Electronic Circular Dichroism Analysis, and Biological Properties. J. Org. Chem. 2017, $82,5574-5582$.

(15) Fruchey, E. R.; Monks, B. M.; Patterson, A. M.; Cook, S. P. Palladium-Catalyzed Alkyne Insertion/Reduction Route to Trisubstituted Olefins. Org. Lett. 2013, 15, 4362-4365.

(16) Gao, Q.; Zheng, B.-F.; Li, J.-H.; Yang, D. Ni(Ii)-Catalyzed Conia-Ene Reaction of 1,3-Dicarbonyl Compounds with Alkynes. Org. Lett. 2005, 7, 2185-2188.

(17) Woźnica, M.; Chaoui, N.; Taabache, S.; Blechert, S. Thf: An Efficient Electron Donor in Continuous Flow Radical Cyclization Photocatalyzed by Graphitic Carbon Nitride. Chem. - Eur. J. 2014, 20, 14624-14628.

(18) Zhao, L.; Lu, X.; Xu, W. Palladium(Ii)-Catalyzed Enyne Coupling Reaction Initiated by Acetoxypalladation of Alkynes and Quenched by Protonolysis of the Carbon-Palladium Bond. J. Org. Chem. 2005, 70, 4059-4063.
(19) Chang, H.-T.; Jayanth, T. T.; Wang, C.-C.; Cheng, C.-H. Cobalt-Catalyzed Reductive Coupling of Activated Alkenes with Alkynes. J. Am. Chem. Soc. 2007, 129, 12032-12041.

(20) Neumann, M.; Zeitler, K. A Cooperative Hydrogen-BondPromoted Organophotoredox Catalysis Strategy for Highly Diastereoselective, Reductive Enone Cyclization. Chem. - Eur. J. 2013, 19, 6950-6955.

(21) Terada, Y.; Arisawa, M.; Nishida, A. Cycloisomerization Promoted by the Combination of a Ruthenium-Carbene Catalyst and Trimethylsilyl Vinyl Ether, and Its Application in the Synthesis of Heterocyclic Compounds: 3-Methylene-2,3-Dihydroindoles and 3Methylene-2,3-Dihydrobenzofurans. Angew. Chem., Int. Ed. 2004, 43, 4063-4067.

(22) Takaki, K.; Beppu, F.; Tanaka, S.; Tsubaki, Y.; Jintoku, T.; Fujiwara, Y. A Novel Cyclodimerization of A,B-Unsaturated Carbonyl Compounds Promoted by Ytterbium Metal. J. Chem. Soc., Chem. Commun. 1990, 516-517.

(23) Cabrera, A.; Le Lagadec, R.; Sharma, P.; Arias, J. L.; Toscano, R. A.; Velasco, L.; Gaviño, R.; Alvarez, C.; Salmón, M. Cyclo- and Hydrodimerization of A,B-Unsaturated Ketones Promoted by Samarium Diiodide. J. Chem. Soc., Perkin Trans. 1 1998, 1, 36093618.

(24) Kapoor, K. K.; Kumar, S.; Ganai, B. A. Zinc Mediated Reductive Dimerization and Cyclization of a,B-Unsaturated Ketones in the Presence of a Catalytic Amount of Mercury(Ii) Chloride. Tetrahedron Lett. 2005, 46, 6253-6255.

(25) Liu, Y.; Dai, N.; Qi, Y.; Zhang, S. Intermolecular Couplization and Cyclization of Chalcones Promoted by Samarium in Dmf. Synth. Commun. 2009, 39, 799-807.

(26) Pitts, M. R.; Harrison, J. R.; Moody, C. J. Indium Metal as a Reducing Agent in Organic Synthesis. J. Chem. Soc., Perkin Trans. 2001, 1, 955-977.

(27) Zhao, G.; Yang, C.; Guo, L.; Sun, H.; Lin, R.; Xia, W. Reactivity Insight into Reductive Coupling and Aldol Cyclization of Chalcones by Visible Light Photocatalysis. J. Org. Chem. 2012, 77, 6302-6306.

(28) Kurpil, B.; Otte, K.; Antonietti, M.; Savateev, A. Photooxidation of N-Acylhydrazones to 1,3,4-Oxadiazoles Catalyzed by Heterogeneous Visible-Light-Active Carbon Nitride Semiconductor. Appl. Catal., B 2018, 228, 97-102.

(29) Kurpil, B.; Kumru, B.; Heil, T.; Antonietti, M.; Savateev, A. Carbon Nitride Creates Thioamides in High Yields by the Photocatalytic Kindler Reaction. Green Chem. 2018, 20, 838-842.

(30) Savateev, A.; Dontsova, D.; Kurpil, B.; Antonietti, M. Highly Crystalline Poly(Heptazine Imides) by Mechanochemical Synthesis for Photooxidation of Various Organic Substrates Using an Intriguing Electron Acceptor - Elemental Sulfur. J. Catal. 2017, 350, 203-211.

(31) Savateev, A.; Kurpil, B.; Mishchenko, A.; Zhang, G.; Antonietti, M. A "Waiting" Carbon Nitride Radical Anion: A Charge Storage Material and Key Intermediate in Direct $\mathrm{C}-\mathrm{H}$ Thiolation of Methylarenes Using Elemental Sulfur as the "S"-Source. Chem. Sci. 2018, 9, 3584-3591.

(32) Su, F.; Mathew, S. C.; Möhlmann, L.; Antonietti, M.; Wang, X.; Blechert, S. Aerobic Oxidative Coupling of Amines by Carbon Nitride Photocatalysis with Visible Light. Angew. Chem., Int. Ed. 2011, 50, 657-660.

(33) Wang, Y.; Li, H.; Yao, J.; Wang, X.; Antonietti, M. Synthesis of Boron Doped Polymeric Carbon Nitride Solids and Their Use as Metal-Free Catalysts for Aliphatic C-H Bond Oxidation. Chem. Sci. 2011, 2, 446-450.

(34) Wang, Y.; Wang, X.; Antonietti, M. Polymeric Graphitic Carbon Nitride as a Heterogeneous Organocatalyst: From Photochemistry to Multipurpose Catalysis to Sustainable Chemistry. Angew. Chem., Int. Ed. 2012, 51, 68-89.

(35) Dontsova, D.; Pronkin, S.; Wehle, M.; Chen, Z.; Fettkenhauer, C.; Clavel, G.; Antonietti, M. Triazoles: A New Class of Precursors for the Synthesis of Negatively Charged Carbon Nitride Derivatives. Chem. Mater. 2015, 27, 5170-5179.

(36) Lau, V. W.-h.; Moudrakovski, I.; Botari, T.; Weinberger, S.; Mesch, M. B.; Duppel, V.; Senker, J.; Blum, V.; Lotsch, B. V. Rational 
Design of Carbon Nitride Photocatalysts by Identification of Cyanamide Defects as Catalytically Relevant Sites. Nat. Commun. 2016, 7, 12165 .

(37) Rodríguez, N. A.; Savateev, A.; Grela, M. A.; Dontsova, D. Facile Synthesis of Potassium Poly(Heptazine Imide) (Phik)/TiBased Metal-Organic Framework (Mil-125-Nh2) Composites for Photocatalytic Applications. ACS Appl. Mater. Interfaces 2017, 9, 22941-22949.

(38) Lau, V. W. h.; Klose, D.; Kasap, H.; Podjaski, F.; Pignié, M. C.; Reisner, E.; Jeschke, G.; Lotsch, B. V. Dark Photocatalysis: Storage of Solar Energy in Carbon Nitride for Time-Delayed Hydrogen Generation. Angew. Chem., Int. Ed. 2017, 56, 510-514.

(39) Kasap, H.; Caputo, C. A.; Martindale, B. C. M.; Godin, R.; Lau, V. W.-h.; Lotsch, B. V.; Durrant, J. R.; Reisner, E. Solar-Driven Reduction of Aqueous Protons Coupled to Selective Alcohol Oxidation with a Carbon Nitride-Molecular Ni Catalyst System. J. Am. Chem. Soc. 2016, 138, 9183-9192.

(40) House, H. O.; Huber, L. E.; Umen, M. J. Empirical Rules for Estimating the Reduction Potential of a,B-Unsaturated Carbonyl Compounds. J. Am. Chem. Soc. 1972, 94, 8471-8475.

(41) Güllü, M. Electrochemical Reduction of Methyl Cinnamate in the Presence of 1,3-Bis(4-Methyl Phenylsulphonyloxy)Propane. Turk J. Chem. 1999, 23, 361-367.

(42) Alston, J. Y.; Fry, A. J. Substituent Effects on the Reduction Potentials of Benzalacetophenones (Chalcones) Improved Substituent Constants for Such Correlations. Electrochim. Acta 2004, 49, 455459.

(43) Hansch, C.; Leo, A.; Taft, R. W. A Survey of Hammett Substituent Constants and Resonance and Field Parameters. Chem. Rev. 1991, 91, 165-195. 